\section{$\underset{\substack{\text { hommes } \\ \text { \& migrations }}}{ }$}

\section{Hommes \& migrations}

Revue française de référence sur les dynamiques

migratoires

1306 | 2014

Ecriture et migration

\title{
Migrations mises en récit ou quand l'espace ne cesse de se (re)construire
}

\section{Amandine Spire}

\section{(apenEdition \\ Journals}

\section{Édition électronique}

URL : http://journals.openedition.org/hommesmigrations/2810

DOI : 10.4000/hommesmigrations.2810

ISSN : 2262-3353

\section{Éditeur}

Musée national de l'histoire de l'immigration

\section{Édition imprimée}

Date de publication : 1 avril 2014

Pagination : 101-107

ISBN : 978-2-919040-27-8

ISSN : $1142-852 X$

\section{Référence électronique}

Amandine Spire, «Migrations mises en récit ou quand l'espace ne cesse de se (re)construire ", Hommes \& migrations [En ligne], 1306 | 2014, mis en ligne le 01 avril 2017, consulté le 19 avril 2019. URL : http://journals.openedition.org/hommesmigrations/2810 ; DOI : 10.4000/ hommesmigrations.2810 


\section{MIGRATIONS MISES EN RÉCIT OU QUAND L'ESPACE NE CESSE DE SE (RE)CONSTRUIRE}

par AMANDINE SPIRE, maître de conférences en géographie, laboratoire CESSMA (UMR 245), université Paris-VII.

Entre l'enquêté et le chercheur, des représentations différentes des étapes et des enjeux d'un parcours migratoire peuvent influer sur la compréhension d'un récit de vie. Au moment de l'entretien, l'espace parcouru et les temporalités de l'ailleurs entrent dans un processus de reconstruction. La mise en mots et la mise en carte d'une histoire migratoire ouvrent la voie à un questionnement sur les catégories et les référents spatiaux en jeu dans les écritures de la migration. Le choix de se raconter est aussi une manière de poursuivre son voyage.

La mise en scène de soi dans les récits migratoires produit une situation d'entretien particulière, durant laquelle le chercheur demande à l'enquêté de se situer au cour d'une narration qui le place en sujet à part entière. Comme le montre Christine Deprez à propos des enjeux de la catégorisation des espaces dans la construction des points de vue, "les espaces s'articulent sur des temporalités, et c'est ce qui permet la mise en récit ${ }^{1}$. Dès lors, l'étude des migrations appelle à mettre en correspondance ces lieux et ces périodes de vie restitués au moment de la situation d'enquête.

La situation d'entretien impose à l'enquêté un regard rétrospectif sur son parcours, reconstruit à l'aide de catégories et de référents mobilisés au cours d'une narration imposée. Comment (se) rendre compte du caractère fragmenté des expériences spatiales et temporelles lors de la mise en écrit tout en considérant l’inévitable mise en ordre des événements propres à la construction même du récit? Assumant les possibles égarements ou les approximations parsemant les histoires de vie, les chercheurs prennent fréquemment le parti de resituer l'histoire migratoire des enquêtés par des récits "bruts" mais aussi par des cartes, malgré les limites d'une telle démarche. Or il s'agit de prendre en considération - et de donner à voir les recompositions spatiales et temporelles qui 
transforment la migration en une trajectoire plus ou moins linéaire d’une expérience pourtant fragmentée et inachevée.

Le propos qui suit émane d'un retour sur des enquêtes de terrain conduites dans le cadre de travaux de géographie portant sur la figure de l'étranger en Afrique de l'Ouest, et plus particulièrement à Lomé (Togo) et à Accra (Ghana)². À travers les récits que j'ai recueillis sur le terrain, les expériences des migrants soulignent l'omniprésence des étapes dans les villes, tantôt pour quelques mois, tantôt pour des années, avec les nombreuses difficultés afférentes (recherche d'emploi, de logement, sentiment d'exclusion, etc.). Les étrangers que j'ai ren-
La représentation que

le migrant détient

de son voyage est donc

constitutive de son itinéraire

reconstruit a posteriori

au cours de la narration produite dans le cadre de l'entretien. contrés à Accra et à Lomé se situent dans des situations d'immobilité, ce qui ne signifie pas forcément la fin du parcours migratoire.

Enfonction desancragesindividuels et au gré des opportunités à venir, les migrants enquêtés ont fréquemment

l'intention de "poursuivre la route" vers un ailleurs jugé prometteur. La migration est alors envisagée ici comme le produit d'un "savoir-circuler", un savoir-faire dans la mobilité ainsi que dans le passage des frontières.

Comment la narration reconstruit-elle un lien entre les multiples espaces-temps de mobilité des migrants enquêtés dans le cadre d'un travail de recherche ? Comment ne pas trahir la chronologie des emplacements successifs lorsque la mémoire réinterprète le passé chez l'enquêté et que l'enquêteur dispose de catégories et de référents spatiaux - et cartographiques - différents de ceux de l'enquêté ? Comment ne pas laisser dans l'ombre le caractère incertain et transitoire des expériences de l'ailleurs constitutives de la figure de l'étranger dans les villes d'Afrique de l'Ouest?

\section{Dire le voyage et l'ailleurs dans des situations transitoires}

L'itinéraire, au même titre que la trajectoire, présuppose un chemin tracé, voulu, ou encore choisi avec préméditation, même si "parler d'itinéraires, c'est parler du départ, du séjour et du retour, même s'il doit être entendu qu'il y eut plusieurs départs, que le séjour fut aussi voyage et que le retour n'a jamais été définitif". La définition de Marc Augé nous rappelle que l'itinéraire n'est pas forcément linéaire : il n'exclut ni les retours ni les va-et-vient. Mais l'idée d'un projet sous-tend le parcours, même si l'individu qui trace son itinéraire en a plus ou moins conscience, comme le souligne Philippe Gervais-Lambony : "Litinéraire est voyage, qui peut comprendre des étapes, et regard porté sur ce voyage." La représentation que le migrant détient de son voyage est donc constitutive de son itinéraire reconstruit a posteriori au cours de la narration produite dans le cadre de l'entretien. Le point de vue est donc situé et en partie déterminé par le contexte d'énonciation à partir duquel s'actualisent les représentations du passé.

La restitution d'un itinéraire migratoire par l'enquêté suggère, par ailleurs, que les pratiques successives de la mobilité ne résultent pas du hasard : parler d'“itinéraire" présuppose un certain degré de maîtrise de la mobilité par le migrant. Au cours des histoires migratoires recueillies, les pratiques rapportées sont ordonnées tout en laissant entrevoir la prise de risque et la saisie d'opportunités dans des espaces de proximité. Les expériences de la migration sur lesquelles nous nous sommes penchées en Afrique de l'Ouest sont empreintes de périodes d'incertitude, d'hésitations, de détours, autrement dit d'errance : 'L'errance est tout autre: elle est mouvement permanent et libre. L'errance n'a, a priori, pas de but ${ }^{5}$." 
Dès lors, nous avons fait le choix de ne pas présupposer un projet migratoire dans l'analyse des itinéraires rapportés par nos enquêtés. Peut-être du fait de la faible capacité sociale et économique des acteurs étudiés, la migration est ici considérée comme une capacité à circuler en jouant sur le potentiel, même faible, de territoires séparés, dans la lignée de travaux sociologiques influencés par ceux d'Alain Tarrius ${ }^{6}$. Sans cesse renouvelés, les systèmes migratoires qui ont retenu notre attention en Afrique de l'Ouest se caractérisent par l'instabilité et le provisoire, supposant nécessairement le "savoir-faire" dans la circulation internationale. Ainsi, il semble incontournable de concevoir la mobilité dans une dimension multiscalaire, mobilisant un répertoire d'action variable selon les individus. Le choix de - et dans - la mobilité dépend de l'élargissement des possibles offert par le mouvement, rejoignant l'idée que la mobilité spatiale pourrait être comparée à une forme de butinage, "qui devient déambulation du hasard et de l'aléatoire mais qui est, en même temps, marque d'appropriation, même précaire et transitionnelle ${ }^{7 "}$. Le hasard n'est pas ici à concevoir comme l'aléatoire, mais plutôt comme la forme d'adaptation des migrants aux crises sociales et économiques que traversent les sociétés ouest-africaines.

\section{L'aventure comme clé du parcours migratoire}

La notion d'aventure permet, dans une certaine mesure, de rendre compte de la variété des parcours, c'est-à-dire des détours et des allées et venues de certains migrants étrangers enquêtés à Lomé ou à Accra. L'aventure renvoie à une forme de migration scandée par des escales plus ou moins longues, le long d'un trajet au but incertain. La consolidation de la catégorie "aventurier" est au centre de la thèse de sociologie d'Anaïk Pian'.
Par ce terme, elle désigne les migrants en transit entre l'Afrique de l'Ouest et l'Europe, c'est-à-dire dans une situation d'entre-deux qui se pérennise timidement, notamment au Maroc. Les aventuriers sont incarnés par des hommes ayant migré seuls, et souvent jeunes. Elle montre que la médiatisation de ces migrants clandestins entraîne un amalgame dans les distinc-

tions entre aventuriers, migrants commerçants et étudiants.

À Lomé comme à Accra, "l'aventure" est d'ailleurs un terme récurrent dans les récits construits par les étrangers eux-mêmes pour décrire leurs expériences migratoires vécues
Il ne sera pas ici question de revenir sur la relation dialectique entre identifications étrangères et sociétés urbaines, mais plutôt de montrer comment cette dialectique de l'ici et de l'ailleurs est au cœur de la mise en écriture de la migration. comme un voyage au long cours marqué par des étapes plus ou moins longues qui permettent de redéfinir liitinéraire. Ce sont donc au cours de ces périodes d'entre-deux, dans des moments de transit que l'on pourrait qualifier d'indéfinis et de prolongés, que nous avons tenté de comprendre les pratiques et les représentations de la mobilité internationale en Afrique de l'Ouest en tant qu'élément central de la définition de la figure de l'étranger en ville. Il ne sera pas ici question de revenir sur la relation dialectique entre identifications étrangères et sociétés urbaines, mais plutôt de montrer comment cette dialectique de l'ici et de l'ailleurs est au cœur de la mise en écriture de la migration.

\section{Dire en mots, dire en cartes}

Nous allons étayer notre propos à l'aide d'un portrait de migrant dressé à partir d'un entretien mené en français, en 2008, à Lomé. Il s'agit de D., que l’on peut considérer comme un aventurier guinéen en 
période de "pause migratoire" à Lomé. Son itinéraire est celui d'un voyage risqué, par la route. Il rejoint, à bien des égards, les conditions de déplacement des candidats clandestins à la traversée du Sahara dépeintes par le journaliste Serge Daniel à partir d'une enquête qu'il mena pendant plusieurs mois à travers différents pays d'Afrique de l'Ouest ${ }^{9}$. Il rapporte les propos tenus par les passeurs face à un groupe prêt à la migration clandestine pour l'Europe, à la gare routière de Lomé : "Il faut oublier vos noms, et savoir que vous êtes des aventuriers. Les aventuriers, ce sont des gens qui vont quelque part, mais qui vont rencontrer des difficultés, des gens qui vont souffrir et qui peuvent donc ne pas arriver à destination ${ }^{10}$." Les conseils donnés par les passeurs avant le départ insistent sur la prise de risque inhérente à la migration. Ce risque est d'autant plus grand qu'il concerne le franchissement du désert et la tentative de franchir frauduleusement les frontières de l'Europe. Il reste

Les conseils donnés par les passeurs avant le départ insistent sur la prise de risque inhérente à la migration. Ce risque est d'autant plus grand qu'il concerne

le franchissement du désert et la tentative de franchir frauduleusement les frontières de l'Europe. néanmoins réel dans les tournures prises par certaines aventures migratoires se déroulant au sein des limites de l'Afrique de l'Ouest.

D. est un Peul de 28 ans originaire d'un petit village de Guinée. Il quitte son village et le travail des champs à l'aube de ses 16 ans en quête d'un emploi rémunéré. Il se rend alors directement dans la capitale de Guinée, à Conakry. Il commence par être cireur de chaussures avant d'entrer dans la sphère des activités domestiques, auprès d'une personne âgée. Peu satisfait de ses conditions de vie, D. raconte qu'il prend la décision de mettre un terme à son séjour dans la capitale guinéenne, où il n'entrevoit pas la possibilité d'améliorer ses conditions de vie. Son récit devient alors un peu confus sur les modalités de son départ, mais ce qui ressort, c'est la nécessité de quitter Conakry pour tenter de trouver mieux ailleurs. À cette époque, l'ailleurs se trouve du côté de Banjul, en Gambie. Pourquoi Banjul ? D. explique ce choix par la présence d'un "frère", une relation que l'on devine assez éloignée de l'enquêté mais qui garantirait un accueil minimum tout en servant de point d'étape vers un ailleurs idéalisé, par-delà le Sahara. L'intention de quitter l'Afrique subsaharienne n'est pas formulée en tant que telle par D., mais elle parcourt l'ensemble de son récit en filigrane.

À Banjul, l'expérience est brève. D. explique qu'il déambule dans les rues pour revendre à l'unité des cigarettes pendant que son "frère" tient la boutique. Visiblement déçu de cette nouvelle condition citadine, D. quitte Banjul au bout de six mois et retourne pour quelque temps dans son village : le récit de cette période traduit la difficulté du retour vécu comme un échec ou plutôt comme une parenthèse forcément courte, une sorte de pas en arrière pour mieux se projeter dans l'ailleurs. D. exprime alors l'idée que sa mise en migration est une histoire qui ne peut plus s'arrêter, au risque d'endosser le rôle du perdant. Rapidement, D. quitte son village natal pour retrouver un oncle au Sénégal. Sur les conseils de ce dernier, il franchit à nouveau une frontière internationale pour se rendre à Bamako. Accueilli par un parent éloigné, il pratique une nouvelle petite activité informelle en devenant vendeur de pain. Au bout de six mois, un nouveau départ s'esquisse. Bien que son activité et la protection de son parent lui confèrent une situation jugée assez confortable, D. ne souhaite pas rester à Bamako. Au bout de neuf mois, il migre pour Lomé muni du contact d'un propriétaire de "cafétéria", c'est-àdire d'un petit restaurant de rue populaire à Lomé, qui pourra l'embaucher dès son arrivée. Ce déplacement s'apparente de nouveau à une mobilité de travail, encadrée par le réseau familial au sens large qui offre un mode d'insertion en ville similaire aux expériences de migration précédentes de $\mathrm{D}$. Depuis son arrivée à Lomé, D. est rentré une fois au village en effectuant un détour par le Mali pour éviter 


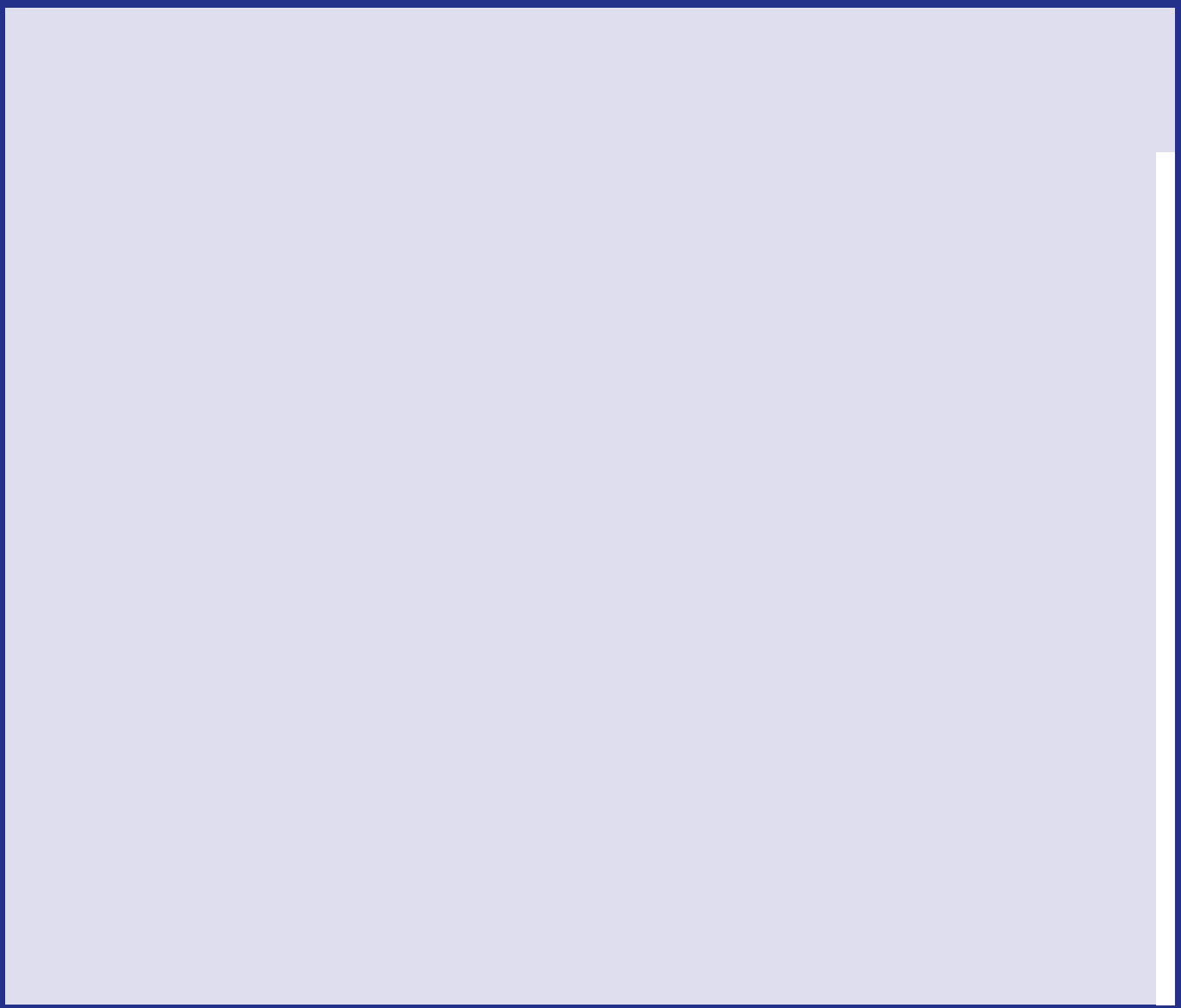

Source : Enquêtes personnelles, 2008

(c) Amandine Spire, Romain Imbach, 2009.

de passer par la Côte d'Ivoire, redoutée tant en raison de son hostilité aux étrangers que pour le coût élevé des péages. Enfin, depuis que D. est à Lomé, un petit frère l'a rejoint, mais D. n'a pas voulu qu'il reste à ses côtés. Il explique qu'il n'était pas en mesure de lui offrir des conditions décentes et il lui a acheté un billet pour le Gabon, où se trouverait un frère plus apte à le prendre en charge. D. n'est pour sa part jamais allé au Gabon tout en envisageant éventuellement de s'y rendre un jour.

\section{Les sous-entendus de la carte}

L'histoire migratoire de D. dure depuis douze ans au moment de l'enquête, si l'on considère que son 
immobilité est partie intégrante d'une trajectoire inachevée. Sa migration est ponctuée d'étapes, de détours et de retours, sur de vastes distances que l'on a considérées propices à un traitement cartographique, présenté ci-contre.

La migration de D. montre bien que les pratiques de la mobilité internationale sont guidées par l'enchaînement d'opportunités au gré des rencontres et de la consolidation d'une "culture de la mobilité" qui rend envisageable le passage de nouvelles frontières. Le fond de carte choisi pour tracer le parcours de D. est celui des États d'Afrique de l'Ouest, membres de la Communauté économique des États de l'Afrique de l'Ouest (Cedeao), à l'exception de la Mauritanie. Dans ma conception de l'espace représenté sous forme cartographique, les frontières jouent un rôle important dans la construction du parcours migratoire. Ces frontières sont sujettes à caution car au fondement de la catégorie juridico-poli-

Le transit migratoire ou la représentation

d'une expérience temporaire dans un espace étranger est dans le même temps synonyme d'une forme de transition de vie que la mise en récit traduit. tique de l'étranger. Cela étant, à aucun moment D. ne mentionne l'importance de ces discontinuités territoriales et politiques dans le récit de ses expériences de l'ailleurs. Et pourtant, la référence à son étrangéité est présente et s'inscrit pleinement dans ses pratiques sociales loméennes.

D. participe, par exemple, à la vie associative de son pays d'origine en se rendant aux réunions mensuelles de l'association de ses ressortissants dans un quartier péricentral afin de recueillir des nouvelles des Guinéens de Lomé et des informations sur l'actualité de son pays. Il fait aussi partie de l'association des ressortissants de sa région natale située au centre-ville et y cotise 500 CFA par mois pour contribuer au fonds de solidarité.

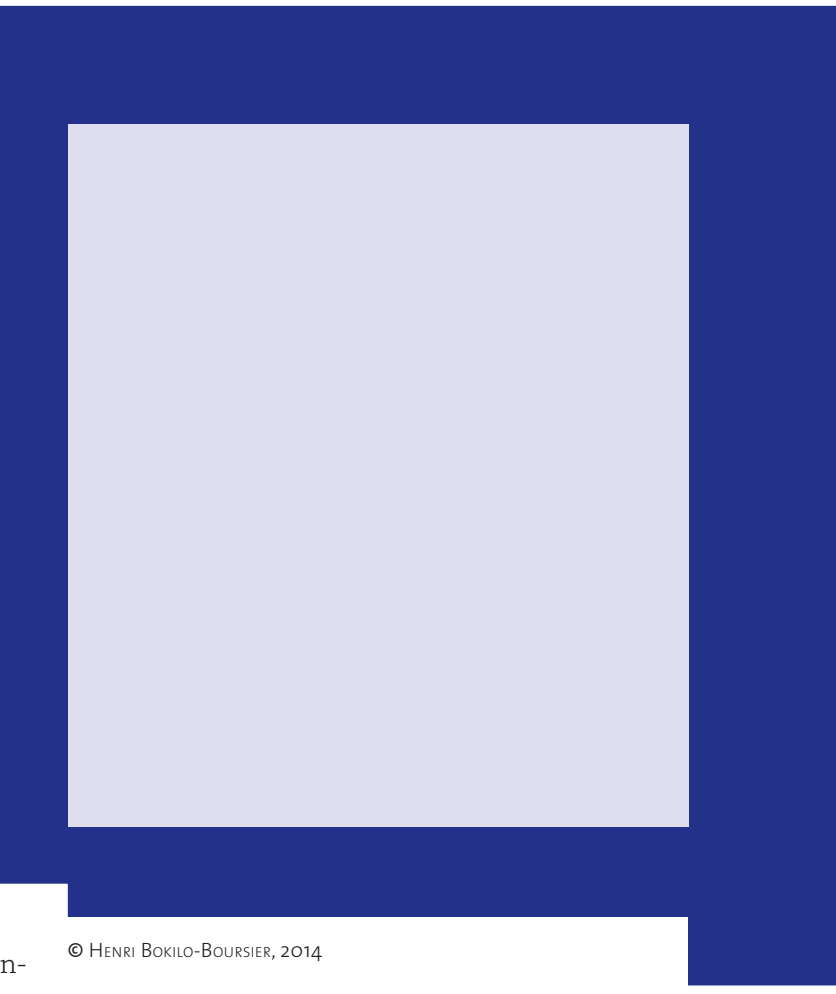

\section{Sentiment d'étrangeté et traversée des frontières}

D. assume donc une posture étrangère à Lomé et se considère comme Guinéen au moment de l'enquête. D'ailleurs, lors des élections présidentielles, il se rend au Consulat pour voter. Il répète à plusieurs reprises qu'il ne souhaite pas rester à Lomé mais qu'il veut rentrer dans "son" pays dès qu'il estimera la situation politique, sociale et économique plus propice. Sa posture étrangère induit aussi des formes de défiance ou de prudence à certaines périodes. En 2005, le Togo, et particulièrement Lomé, connaît une vive période de troubles durant laquelle les étrangers sont pris pour boucs émissaires selon un schéma assez classique ${ }^{11}$. En tant qu'employé de cafétéria, secteur connu et reconnu comme un domaine d'activité de prédilection des 
étrangers, et spécialement des Guinéens, D. a dû se mettre en retrait au plus fort des mobilisations politiques. Préventivement, il a d'ailleurs fermé sa boutique pendant trois semaines afin d'éviter d'être visible et repéré par les opposants. Cette période particulière de son séjour à Lomé est le produit de sa position d'étranger et de son expérience migratoire. La mise en carte de son itinéraire migratoire donne à voir la répétition du passage des frontières bien que D. n'insiste jamais sur ce point. Les frontières qu'il évoque en creux dans son récit sont plutôt les distances sociales et culturelles instrumentalisées dans les rapports de force politiques locaux.

Un autre point met en exergue les limites de la transcription de l'expérience de D. à travers la mise en carte. Les grandes villes de la sous-région sont localisées pour situer le voyage de D., avec l'idée que l'acquisition d'une initiation à la vie urbaine se réalise au gré de la migration pour un migrant d'origine rurale. Cependant, une certaine confusion s'instaure dans le récit de $\mathrm{D}$. quand il évoque son séjour à Bamako. Au sujet de la capitale malienne, il explique: "Là-bas, c'était encore un village, l'oncle voulait que je reste, mais moi je voulais aller en ville." Étrangement, la capitale du Mali ne correspond pas pour D. à l'expérience urbaine et la quête de l'urbain serait même une raison pour un nouveau départ. Cette représentation est-elle influencée par la localisation de la boutique de l'oncle et des lieux de distribution qui pourraient se situer dans des villages péri-urbains ? Est-ce que D. entend par ville une capitale littorale comme Conakry, la ville où il a appris à devenir citadin ?

Ce détail du récit de $\mathrm{D}$. explicite les différentes représentations et catégorisations qu'enquêté et enquêteur détiennent et la manière dont la mise en mots et en cartes transforme ou réduit la complexité des expériences sociales qui définissent l'itinéraire migratoire. Finalement, à travers le portrait de D., le retour sur la manière dont s'écrit la migration met en lumière la confrontation des représentations de l'ailleurs dans une situation d'entretien, ainsi que les différentes représentations de la relation dialectique de l'ici et de l'ailleurs qui définit la posture d'étranger. Plus qu'une rupture entre un espace de départ et une société d'accueil, le déplacement migratoire induit la constitution d'un espace de vie marqué par un faisceau de relations où l'identification au groupe fluctue ${ }^{12}$. À ce titre, l'histoire migratoire devient bien une histoire de vie.

\section{Le voyage inachevé...}

Les parcours migratoires restitués par les enquêtés rencontrés à Lomé et à Accra donnent à voir des itinéraires caractérisés par le mouvement et l'immobilité en différents lieux, le plus souvent urbains : ce sont des temps de transit, de l'attente... Le transit migratoire ou la représentation d'une expérience temporaire dans un espace étranger est dans le même temps synonyme d'une forme de transition de vie que la mise en récit traduit. Dans les situations d'enquête que nous avons provoquées à Lomé comme à Accra, l'espace-temps de l'entretien fait souvent partie intégrante du parcours inachevé de l'enquêté. Quel poids alors accorder aux projections que l'enquêté construit pour donner du sens aux étapes de son parcours? Comment donner du sens aussi aux différentes pratiques sociales dans la migration, dès lors que l'on considère l'incessante reconstruction du sujet à travers le récit? 